\title{
Surgical management of a case of subcutaneous abscess caused by Staphylococcus aureus in adult White Fulani cow
}

${ }^{1}$ Sogebi,E. A. O., ${ }^{1}$ Talabi, O. A., ${ }^{1}$ Salami, I. O., ${ }^{2}$ Babalola, S. A., ${ }^{3}$ Eze C. A.

${ }^{1}$ College of Veterinary Medicine, Federal University of Agriculture, Abeokuta.

Ogun state. Nigeria. ${ }^{2}$ Department of Veterinary Surgery \& ,Micheal Okpara University of Agriculture, Umudike. ${ }^{3}$ Department of Veterinary Surgery \& Radiology, University of

Nigeria, Nsukka.

Corresponding author: sogebieao@funaab.edu.ng

\section{Abstract}

A White Fulani cow whose record reveals three-year old, weighing about $350 \mathrm{~kg}$ belonging to DUFARMS of the Federal University of Agriculture, Abeokuta was presented with a swelling on the left flank. Clinical evaluation of the animal revealed the following: rectal temperature, $38.2^{\circ} \mathrm{C}$, heart rate, $60 \mathrm{bpm}$ and respiratory rate, $35 \mathrm{bpm}$. A rectangular shaped mass, soft and warm to touch was palpated on the left flank. Pus was aspirated from the swelling with a sterile needle and syringe, and taken for biogram. Staphylococcus aureus was isolated from the aspirate which was sensitive to antibiotic, enrofloxacin, ciprofloxacin and gentamycin (Table 1). Total surgical extirpation of the encapsulated abscess was carried out aseptically under sedation and local anaesthesia and managed chemotherapeutically using antimicrobial, analgesic, anti-inflammatory agents post operatively.

Keywords: White Fulani cow, Subcutaneous abscess, Staphylococcus aureus, Enrofloxacin, surgical extirpation,

\section{Introduction}

Abscess is a cavity caused by tissue destruction, usually of infection, filled with pus and surrounded by inflamed tissue (Sasty and Rama, 2004; Blood et al., 2007). Abscesses are characterized by appearance of circumscribed nodules which gradually develop into closed abscess containing bloody tinged pus or pure creamy pus along the course of lymphatic vessels of the forelimbs, abdomen and thigh (Sayed et al., 2007). The subcutis is the commonest site of an abscess (Sasty and Rama, 2004; Blood et al., 2007). Subcutaneous abscesses are localized suppurative inflammation limited by a wall of granulation tissue. They are usually painful, tender, and fluctuant red nodules, often surrounded by a rim of erythematous swelling. They are typically polymicrobial, containing bacteria that constitute the normal regional skin flora, often combined with organisms from adjacent mucous membrane (Brook and
Frazier, 1990).

Numerous organisms can cause abscess but Staphylococcus aureus is the most common in sheep and goats (Tadayon et al., 1980). The modes of transmission include: direct implantation (penetrating trauma with a contaminated object) or break in integrity of the skin; spread from an established contagious infections; dissemination via lymphatic or haematogenous route from a distant site and migration from a location where there are resident flora into adjacent normally sterile areas because natural barriers are disrupted (Chamberline and Brueggemann, 1997; Fitzgerald et al., 2000). One of the causes of abscess is infection in cattle caused staphylococcus aureus spread to the lymphatics.

Subcutaneous abscess can be diagnosed using ultrasonography, radiography, physical examination, aspiration of pus from the affected part, culture and Gram staining techniques with direct microscopy 


\section{Surgical management of subcutaneous abscess}

(Scott et al., 2001). Systemic antibiotics are often used for treatment according to the organism causing the abscess and its sensitivity to them.

Effective treatment of abscesses is through thorough evacuation of the pus by surgical drainage and probing the cavity to break up loculations loculi. Covering the surgical site with a dry dressing is usually the easiest and most effective treatment of the wound (Llera and Levy, 1995), although some clinicians pack it with gauze or suture it closed however some clinicians pack the wound with gauze while others suture and close the wound. There is dearth of information on the occurrence and common causes of cutaneous abscess in cattle abscess on this farm. This work is a documentation of a case of abscess which was effectively managed by a combination surgical procedure and therapy.

\section{Materials and Methods}

Background/Case History: A three-year old, according to the record on the farm, White Fulani cow weighing about $350 \mathrm{~kg}$ was presented at the Veterinary Teaching Hospital of the Federal University of Agriculture, Abeokuta with the history of swelling on the left lateral side of the abdomen towards the ventral part of the lumber region. The swelling was about two months in existence.

Clinical Examination/signalment: Clinical Examination revealed a rectal temperature of $38.2^{\circ} \mathrm{C}$, heart rate of 60 beats per minute and respiratory rate of 35 beats per minute. Physical examination revealed normal mucous membrane of the eye and the buccal cavity and presence of a rectangular shaped mass on the left flank. The animal had no pain response on palpation and the cow was active and alert.

Sample Collection: The swelling was cleaned with gauze soaked in chlohexidine ${ }^{\circledR}$, and about $500 \mathrm{mls}$ of insissipated pus exudate was aspirated from the swelling in all when measured in a measuring cylinder serially using a $21 \mathrm{G}$ needle and $20 \mathrm{ml}$ syringe, stored in ice bucket and submitted to Microbiology laboratory for culture and sensitivity. However, there was no appreciable regression in the size of the swelling necessitating a surgical excision. The excised mass was found to be localized pus which weighed about $350 \mathrm{~g}$.

Isolation of sample: A sterile inoculating wire loop was used to streak the pus sample directly on a freshly prepared blood agar and incubated aerobically at $37^{\circ} \mathrm{C}$ for $24 \mathrm{hr}$. Suspected colonies were sub cultured on nutrient agar to obtain pure culture. Characteristic colonies were identified by conventional methods, including Gram stain, catalase test, haemolysis, and anaerobic utilization of glucose and mannitol, and coagulase test (Miriam and Rosalia, 2004).

Antibiotic susceptibility test: The disk diffusion method described by Kirby-bauer was used and nine (9) commonly used antibiotics were tested.

Procedure: The pure bacterial isolate from 18-24hr old culture plate was spread evenly on a freshly prepared Mueller-Hinton agar plate and then paper disc containing specific concentration of antibiotics are placed and incubated at $37^{\circ} \mathrm{C}$ overnight. The diameter of zone of inhibition was measured and result read from the Kirby Bauer chart as sensitive, intermediate or resistant (Quinn et al., 2004).

Surgical management and procedure: The surgical procedure was performed on the field where the strict aseptic surgical condition could not be achieved. However universal precautions to minimize infection were applied by clipping of the surgical site with new razor blade and scrubbed with 
chlorhexidine ${ }^{\circledR}$. Venous access was secured at the jugular vein for administration of fluid, lactated ringers solution during the procedure at a maintenance flow rate of $40 \mathrm{ml} / \mathrm{kg} /$ day (Linklater, 2013) and the animal was sedated using xylazine ${ }^{\mathbb{B}}$ at the rate of $0.02 \mathrm{mg} / \mathrm{kg}$ while local anaesthesia was achieved with $2 \%$ lidocaine hydrochloride ${ }^{\circledR}$ using the inverted 'L' block technique, the lidocaine was infiltrated from the T13 caudally to S7 spinal bone then infiltrated down towards the udder terminating at the ventral part of the abdomen.

A semi-circle incision was performed around the swelling with a size 3 surgical blade and using the blunt edge of the blade, a blunt dissection was done slowly and carefully between the skin and the capsule of the abscess. The blood vessels, branches of the lumber vein, encountered were ligated (2-clamp method) and severed to enable a complete detachment of the abscess while hemostasis of the small blood vessels was done by pressure and crushing using mosquito forceps (figure 1). Then dissection of the capsule was continued until complete excision of the abscess was achieved. The abscess weighing about $350 \mathrm{~g}$ was taken to pathology laboratory for histopathology.

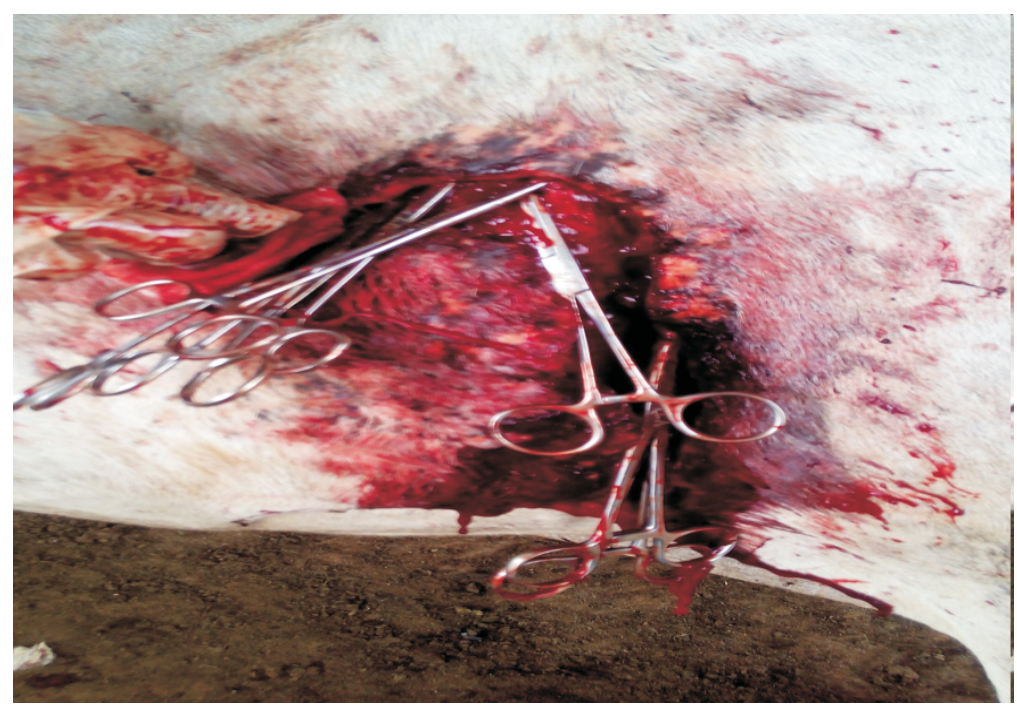

Figure1: Showing control of haemorrhage during surgery

The wall of the abdominal muscle was flushed with normal saline (Figure 2) (Bucknall and Ellis, 1984) and packed with sterile gauze. The subcutaneous tissue and fascia were then sutured with chromic catgut in a simple continuous suture pattern while the skin was closed with silk in a simple interrupted suture pattern leaving a little edge of the sterile gauze outside (Llera and Levy, 1995). 


\section{Surgical management of subcutaneous abscess}

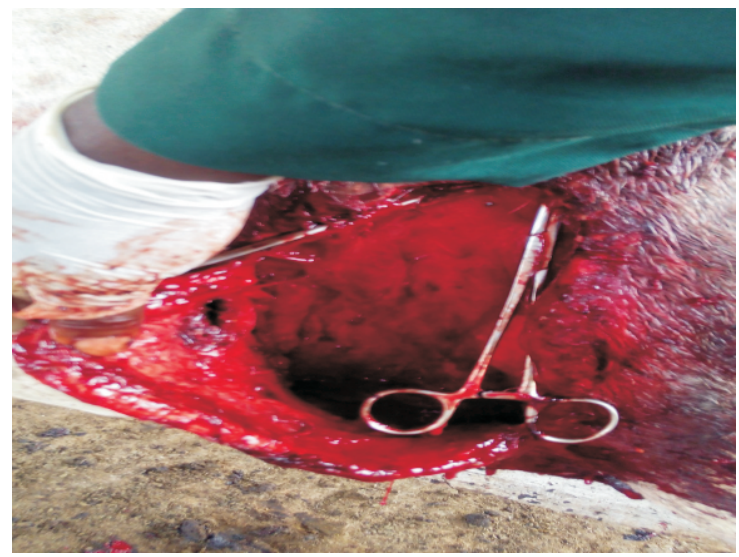

Figure 2: Showing the wall of the abdomen after removal of the insissipated pus

Post-operative management: Twenty (20) $\mathrm{ml}$ of Penicillin-streptomycin was infiltrated at the site of operation, and Penicillin-streptomycin at 20,000 $\mathrm{IU} / 20 \mathrm{mg} / \mathrm{kg}$ bodyweight $2 \mathrm{mg} / \mathrm{kg}$ of Flunixin and $10 \mathrm{mg} / \mathrm{kg}$ of Multivitamin were administered intramuscularly for five days with hypodermal needle and syringes. The cattle was then hospitalized on the farm separate from the other cattle, feed daily on grass and concentrate and water were given ad libitum.

The gauze was removed day 3 post-surgery and the wound was daily infused thereafter with honey for another 7 days while Povidone iodine was topically applied to the surgical area until healing. . The skin suture was removed 2-weeks post-surgery.

\section{Results}

The pus sample revealed the presence of Staphylococcus aureus after culture and gram staining (Figure 3). The result of the sensitivity test (Table 1) indicated that the organism was sensitive to Ciprofloxacin, Pefloxacin, Gentamycin and Chloramphenicol but resistant to A u g m entin, Streptom ycin, Sulphamethoxazole, Sparfloxacin and Amoxicillin.

This result prompted the line of medication to be changed from Pen-Strep to Enrofloxacin. Enrofloxacin was administered at $10 \mathrm{mg} / \mathrm{kg}$ intramuscularly for 5 days and the animal showed great improvement, the incision line progressively apposing (Figure 4).

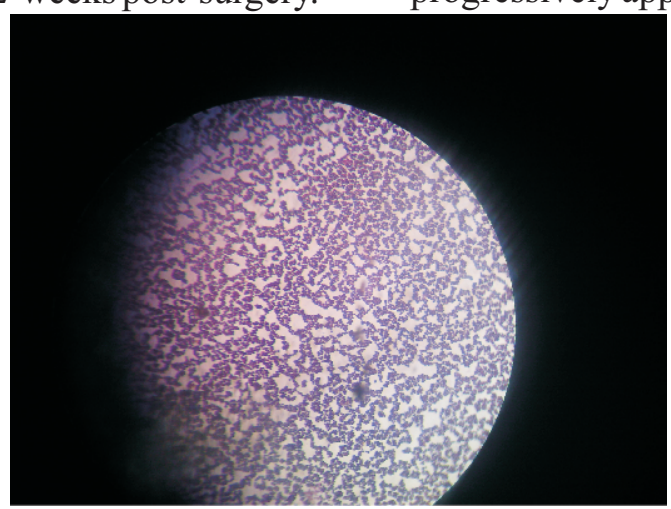

Figure 3: Picture showing Staphylococcus aureus under the microscope 
Sogebi., Talabi., Salami., Babalola. and Eze

Table 1: Result of the sensitivity test

\begin{tabular}{ll}
\hline Antibiotics $(\boldsymbol{\mu g})$ & Result \\
\hline Ciprofloxacin & Sensitive \\
Pefloxacin & Sensitive \\
Gentamycin & Sensitive \\
Chlora mphenicol & Sensitive \\
Augmentin & Resistant \\
Streptomycin & Resistant \\
Sulphamethoxazole & Resistant \\
Sparfloxacin & Resistant \\
Amoxicillin & Resistant \\
\hline
\end{tabular}

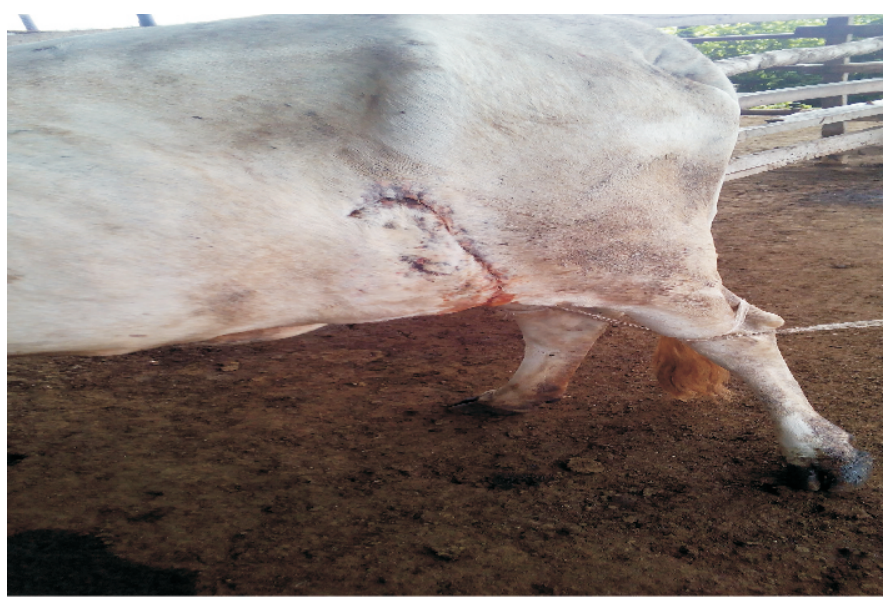

Figure 4: Picture showing the animal five days post-surgery.

The pathology result of the affected subcutaneous tissue revealed diffused degeneration and caseous necrosis of the myofibres characterized by pyknosis, karyorrhexis and kuryolysis of the myofibres (white arrow). There were multiple foci of bacterial colonies within the necrotic areas.

Neutrophils were embedded in the caseous material and necrotic areas surrounded by mononuclear cells (macrophages) and fibroblast (green arrow) (Figure 5).

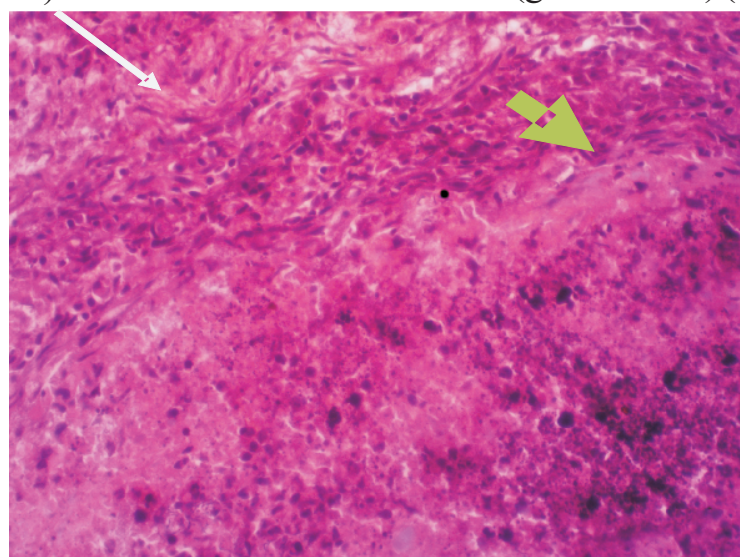

Figure 5: Photo-microgragh of the muscle showing caseous necrosis and bacterial colonies surrounded by fibrous connective and mononuclear cells (arrowed) 


\section{Discussion}

Subcutaneous abscess is a common condition in cattle. It is a chronic condition that can arise slowly and characterized by one or more abscesses on different areas of the skin. The condition in the present study was diagnosed by physical examination (palpation) and aspiration, while culture and sensitivity defined the contaminant and line of therapy. Radiography and ultrasonography could also be an alternative in the diagnosis of subcutaneous abscess (Scott et al., 2001) which was considered in this case but the facility was not available.

The insissipated pus was taken to the pathology laboratory for further diagnosis to rule out any form of tumor involvement in this case. The isolation of $S$. aureus from subcutaneous abscesses of cattle is in agreement with the results of Tadayon et al., (1980), they showed that the most frequent bacteria that were isolated from subcutaneous abscesses of sheep and goat belong to three genera, namely Corynebacterium, Staphylococcus and Streptococcus. Penicillin-streptomycin been a broad spectrum antimicrobial agent was infiltrated to prevent secondary bacterial infection. It was also administered through the intramuscular route to help prevent against systemic infection which could delay or prevent healing depending on the extent of infection. This line of treatment was changed for a more appropriate therapy when the organism cultured was sensitive to Ciprofloxacin and Enrofloxacin. Flunixin was administered as analgesic and anti-inflammatory drug to manage pain and inflammatory changes, which are factors responsible for delay wound healing. Multivitamin was used as an appetite stimulant, these are supportive therapy necessary to facilitate healing process in the patient. The surgical site was flushed aggressively with normal saline after removal of the mass to ensure sterility of the tissue while maintaining the integrity of the tissue which could be compromised if any disinfectant is used. The topical application of povidone iodide until wound healing prevent contamination of the wound from direct contact since the animal was left on the farm (field environment) throughout the period of wound healing. Total extirpation was possible in this case because the abscess was accessible with a well defined capsule and no surrounding cellulitis. This is in agreement with the finding of Flower, 1998.

In conclusion, subcutaneous abscess can be treated effectively by surgery and the use of appropriate antimicrobial agents.

\section{References}

Blood, D. C., Studdert, V. P. and Gay, C. C. 2007. Saunders Comprehensive Veterinary Dictionary, 3rd ed., Saunders Elsevier. London. 6, 1719.

Brook, I. and Frazier, E. H. 1990. Aerobic and anaerobic bacteriology of wounds and cutaneous abscess. Archives Surgery. 125. Pp. 1445-51.

Bucknall and Ellis. 1984. Wound healing for surgeons. London, Bailliere Tindall. Pp. 344

Chamberline, N. R. and Brueggemann, S. A. 1997. Characterisation and expression of fatty a c i d modifying enzyme produced by Staphylococcus epidermidis. Journal of $M$ edical Microbiology. 46. Pp. 693-697. doi: 10.1099/00222615-46-8-693

Fitzgerald, J. R., Hartigan, P. J., Meaney, W. J. and Smyth, C. J. 2000. Molecular population and virulence factor analysis of Staphylococcus aureus from bovine intramammary infection. Journal of 
Applied Microbiology . 88( 6). Pp. 1028-1037.

Flower, M. E. 1998. Medicine and surgery of South American camelids: Llama, Alpaca, Vicuno, Guanaco. $2^{\text {nd }}$ edition, Iowa State University Press, Iowa, U.S.A.

Linklater, A. 2013. Maintenance fluid plan ; Fluid therapy : The Merck Veterinary Manual. Pp.1-2.

Llera, J. L. and Levy, R. C. 1995. Treatment of cutaneous abscess: a double-blind clinical study. Annual. Emergency Medicine. 14. Pp.15-9

Miriam, O. I., Rosalia. F. 2004. Microbiological quality of Port Salute Argentina cheese stored at two temperature treatments, Lebensm. Wiss. Technology. 37. Pp. 739-748.

Quinn, P. J., Carter, M. E., Markey, B. K. and Carter, G. R. 2004. Clinical veterinary microbiology. London, Mosby International -year book 82 Chapter 6. Management Practices Associated With Pathogen Specific Incidence Rate of Clinical Mastitis Europe limited. Pp 628.

Sasty, G. A. and Rama, P. R. 2004.
Veterinary pathology. 7th edition, Satish Kumar Jain, New Delhi, India.

Sayed, S. M., Rateb, H. Z., Arafa, M. I., Abdel-Hafeez M. M. and Amer, A. A. 2007. Field study on buffalo oedematous skin disease in Assiut governorate. Assiut Veterinary Medicine Journal. 53 (114). Pp.1892006.

Scott, D. W., Miller, W. H. and Griffin, C. E. 2001. Muller \& Kirk's Small Animal Dermatology, 6th ed., W.B. Saunders, Philadelphia, PA. Pp. 1125-1184.

Tadayon, R. A., Cheema, A. H. and Muhammed, S. I. (1980). Microorganisms associated with abscesses of sheep and goats in south of Iran. American Journal of Veterinary Research. 41 (5). Pp. 798802. 